\title{
Weed Control, Environmental Impact and Profitability of Pre-Plant Incorporated Herbicides in White Bean
}

\author{
Nader Soltani $^{{ }^{*}}$, Robert E. Nurse ${ }^{2}$, Christy Shropshire ${ }^{1}$, Peter H. Sikkema ${ }^{1}$ \\ ${ }^{1}$ University of Guelph Ridgetown Campus, Ridgetown, Canada; ${ }^{2}$ Agriculture and Agri-Food Canada, Harrow, Canada. \\ Email: ${ }^{*}$ nsoltani@ridgetownc.uoguelph.ca
}

Received April 16 ${ }^{\text {th }}, 2012$; revised May $16^{\text {th }}, 2012$; accepted May $23^{\text {rd }}, 2012$

\begin{abstract}
Three field experiments were conducted over a three-year period (2009 to 2011) to evaluate various preplant incorporated (PPI) herbicides in white bean in Ontario, Canada. There was minimal visible injury in white bean for any of the treatments evaluated. The control of AMARE, AMBEL, CHEAL, SETVI, and SINAR ranged $91 \%-93 \%, 13 \%-21 \%$, $56 \%-92 \%, 96 \%-98 \%$, and $16 \%-24 \%$ with pendimethalin, $93 \%-99 \%, 34 \%-43 \%, 65 \%-99 \%, 95 \%-100 \%$, and $20 \%-47 \%$ with trifluralin, $66 \%-77 \%, 61 \%-76 \%, 64 \%-86 \%, 94 \%-99 \%$, and $52 \%-74 \%$ with EPTC, $92 \%-98 \%$, $27 \%-48 \%, 28 \%-76 \%, 96 \%-99 \%$, and $33 \%-59 \%$ with s-metolachlor, $98 \%-99 \%, 50 \%-72 \%, 33 \%-88 \%, 98 \%$ $99 \%$, and $56 \%-78 \%$ with dimethenamid, and $98 \%-100 \%, 72 \%-84 \%, 97 \%-100 \%, 77 \%-88 \%$, and $98 \%-100 \%$ with imazethapyr, respectively. Weed density and dry weight reduction were similar to visible weed control. Imazethapyr applied PPI at the $75 \mathrm{~g} \cdot$ ai $\cdot \mathrm{ha}^{-1}$ provided yield equivalent to the weed-free check in 2009 and 2011 . All other herbicide treatments had significantly lower yield than weed-free check. All herbicide treatments except pendimethalin at 1080 $\mathrm{g} \cdot \mathrm{ai}^{\mathrm{h}} \mathrm{ha}^{-1}$, EPTC at $4400 \mathrm{~g} \cdot \mathrm{ai} \cdot \mathrm{ha}^{-1}$, and imazethapyr at $75 \mathrm{~g} \cdot \mathrm{ai} \cdot \mathrm{ha}^{-1}$ had yield equivalent to the weed-free check in 2010. Based on environmental impact (EI) analysis, the herbicide program with the lowest environmental risk was imazethapyr followed by dimethenamid-p, trifluralin, s-metolachlor, EPTC, and then pendimethalin. Economic analysis of herbicide treatments evaluated indicates that imazethapyr had the greatest positive impact on profit margins, followed by dimethenamid-p and EPTC, followed by s-metolachlor and trifluralin and then pendimethalin.
\end{abstract}

Keywords: Dimethenamid; EPTC; Imazethapyr; Navy Bean; Pendimethalin; S-Metolachlor; Trifluralin

\section{Introduction}

North America is the largest producer of white bean (Phaseolus vulgaris L.) in the world [1]. In 2010, Ontario growers planted approximately 34,000 hectares and produced 82,600 tonnes of white bean with a farm gate value of $\$ 55,200,000$ [2]. White bean has short stature and is not a strong competitor with weeds. Weed interference can result in large yield losses, reduce harvest efficiency, and may stain white bean resulting in reduced market value [3-5]. Troublesome weeds in white bean production in Ontario include common lamb's-quarters (Chenopodium album L.), redroot pigweed (Amaranthus retroflexus L.), velvetleaf (Abutilon theophrasti Medic), wild mustard (Sinapis arvensis L.), common ragweed (Ambrosia artemisiifolia L.), Solanum spp. (annual nightshades), and green foxtail (Setaria viridis (L.) Beauv.) [6]. More research is needed to identify herbicides that have an adequate margin of crop safety, provide consistent weed control, have low environmental

${ }^{*}$ Corresponding author. impact and maximize dry bean yield and net returns to white bean growers in Ontario.

Pendimethalin is a dinitroaniline selective herbicide that can control annual grasses such as barnyardgrass (Echinochloa crusgalli (L.) Beauv.), smooth crabgrass (Digitaria ischaemum (Schreb) Muhl.), large crabgrass (Digitaria sanguinalis (L.) Scop), fall panicum (Panicum dichotomiflorum Michx., giant foxtail (Setaria faberii Herrm.), S. viridis, yellow foxtail (Setaria glauca (L.) Beauv.), and certain annual broadleaf weed such as $C$. album and $A$. retroflexus including acetolactate synthase and triazine-resistant biotypes [6,7].

Trifluralin is a dinitroaniline herbicide that controls several annual grasses including Setaria, Digitaria, Echinochloa, and Panicum spp. and some broadleaved weeds such as C. album and A. retroflexus, including acetolactate synthase and triazine-resistant biotypes $[6,7]$.

EPTC is a thiocarbamate herbicide that provides control of a number of annual grasses such as Setaria spp., $D$. sanguinalis, E. crusgalli, P. dichotomiflorum, and yellow nutsedge (Cyperus esculentus L.) [6,7]. EPTC can also 
control some broadleaf weeds such as C. album, A. retroflexus and $S$. media including acetolactate synthase and triazine-resistant biotypes [6].

S-metolachlor and dimethenamid-p are chloroacetanilide herbicides that can provide effective control of a number of annual grasses such as $S$. faberii, S. viridis, $S$. glauca, E. crusgalli, D. ischaemum, P. dichotomiflorum and $P$. capillare $[6,7]$. They also provide partial control of some small-seeded broadleaved weeds such as Solanum spp., C. album, and A. retroflexus.

Imazethapyr is an imidazolinone herbicide that controls several annual grass and broadleaved weeds including Setaria spp, E. crus-galli, P. capillare, Polygonum convolulus L. (wild buckwheat), Polygonum persicaria L. (ladysthumb), C. album, S. arvensis, Solanum spp., A. retroflexus, A. artemisiifolia, and $A$. theophrasti including triazine-tolerant biotypes [6-10].

Many factors are considered when selecting a weed management strategy including crop safety, weed spectrum, crop rotation, and costs. The environmental impact (EI) of herbicides should also be considered when making weed management decisions. By using lower herbicide application doses, the EI of weed control is reduced. One method to assess the EI of a pesticide is with the environmental impact quotient (EIQ) [11]. The EIQ uses toxicity (chronic, dermal, fish, bird, arthropod, and bee), leaching and surface loss potential, and soil and plant half-life to estimate the relative potential risk of pesticide active ingredients. The EIQ has been used to compare environmental risk of different pesticides and/or production systems [12-14]. The EIQ was designed to provide growers and other decision makers with a single number that indicates the magnitude of relative risk. The EI of a particular pesticide treatment is obtained by multiplying the EIQ by the application rate. A higher EI indicates a greater risk of detrimental impact [11].

Identification of herbicides that provide consistent effective weed control, have low environmental impact and maximize dry bean yield and net returns would be of benefit to Ontario dry bean producers. There is little information on the relative efficacy, environmental impact and profitability of pendimethalin, trifluralin, EPTC, s-metolachlor, dimethenamid-p, and imazethapyr applied preplant incorporated (PPI) in white bean under Ontario environmental conditions.

The objectives of this study were to evaluate the efficacy, environmental impact and profitability of pendimethalin, trifluralin, EPTC, s-metolachlor, dimethenamid-p, and imazethapyr applied PPI in white bean.

\section{Materials and Methods}

Three field trials were conducted at the Huron Research Station, Exeter, Ontario in 2009 to 2011. The soil was a Brookston clay loam (Orthic Humic Gleysol, mixed, mesic, and poorly drained) with $44 \%$ sand, $33 \%$ silt, $23 \%$ clay, $4.1 \%$ organic matter and $\mathrm{pH}$ of 7.9 in $2009 ; 32 \%$ sand, $40 \%$ silt, $28 \%$ clay, $4.5 \%$ organic matter and $\mathrm{pH}$ of 7.8 in 2010 , and $35 \%$ sand, $43 \%$ silt, $22 \%$ clay, $4 \%$ organic matter and pH of 7.8 in 2011. Seedbed preparation at all sites consisted of autumn moldboard plowing followed by seedbed preparation and herbicide incorporation with a S-tine cultivator with rolling basket harrows in the spring.

The experiment was arranged in a randomized block design with treatments replicated four times. Treatments are shown in Tables 1-5. Each plot was $3.0 \mathrm{~m}$ wide and $10 \mathrm{~m}$ long and consisted of four rows of "T9905" white bean spaced $0.75 \mathrm{~m}$ apart. White bean was planted at a rate of 250,000 seeds $\cdot \mathrm{ha}^{-1}$ on May 22, 2009, May 25, 2010 and June 3, 2011.

Herbicide treatments were applied using a $\mathrm{CO}_{2}$-pressurized backpack sprayer calibrated to deliver $200 \mathrm{~L} \cdot \mathrm{ha}^{-1}$ at $240 \mathrm{kPa}$. The boom was $1.5 \mathrm{~m}$ long with four ultra-low drift nozzles (ULD120-02, Hypro, New Brighton, MN) spaced $50 \mathrm{~cm}$ apart. The surface area sprayed was the center $2.0 \mathrm{~m}$ of each plot by $10.0 \mathrm{~m}$ in length. There was a $1.0 \mathrm{~m}$ unsprayed area between adjacent plots. Preplant incorporated herbicides were applied 1 - 2 days before planting and were immediately incorporated into the soil with two passes (in opposite directions) of an S-tine cultivator with rolling basket harrows. Weed-free plots were maintained weed free during the growing season by hand hoeing as required.

White bean injury and weed control were visually estimated on a scale of 0 (no injury/control) to $100 \%$ (complete plant death) 1 and 4 weeks after crop emergence (WAE), and 4 and 8 WAE, respectively. Weed density and dry weight were evaluated $8 \mathrm{WAE}$ by counting and cutting plants at the soil surface in two $0.5 \mathrm{~m}^{2}$ quadrats per plot and separating by species. Plants were dried at $60 \mathrm{C}$ to constant moisture and then weighed. White bean was considered mature when $90 \%$ of the pods in the weed-free check had turned from green to a golden colour. Beans were harvested from each plot with a small plot combine, weight and moisture were recorded, and yields were adjusted to $18 \%$ moisture.

Data were analyzed using PROC MIXED in SAS 9.2. Herbicide treatment was considered a fixed effect, while environment (year), environment by treatment interaction, and replicate nested within environment were considered random effects. Significance of fixed effects were tested using F-tests and random effects were tested using a Z-test of the variance estimate. Environments were combined for a given variable if the environment by treatment interaction was not significant. The UNIVARIATE procedure was used to test data for normality and homogeneity of variance. Any treatment assigned a value of zero (weedy check for injury and weed control; weed- 
free check for injury, weed density and dry weights) was excluded from the analysis. However, all values were compared independently to zero to evaluate treatment differences with the weedy and/or weed-free checks. To satisfy the assumptions of the variance analyses, weed control for A. retroflexus and C. album (4 WAE only) and $S$. viridis were arcsine square root transformed, and all weed density and dry weights were log transformed. Treatment comparisons were made using Fisher's Protected LSD at a level of $\mathrm{P}<0.05$. Data compared on the transformed scale were converted back to the original scale for presentation of results.

\subsection{Environmental Impact}

The EIQ values of pendimethalin, trifluralin, EPTC, s-metolachlor, dimethenamid-p, and imazethapyr were obtained from Kovach et al. [11]. The quantity of herbicide applied was multiplied by the EIQ to determine the EI.

\subsection{Profit Margin}

A partial economic analysis was conducted to determine the profit margins for each herbicide treatment. The margins were calculated using white bean yields, the average annual price received by white bean growers in Ontario from 2009 to 2011 (Ontario White Bean Producers' Marketing Board) and herbicide costs in 2009-2011 reported by AGRIS Co-operative Ltd [15]. The profit margin per plot was calculated and data were analyzed using PROC MIXED in the same manner as above, and both the weedy and weed-free checks were excluded from analysis. Data were square root transformed to satisfy the assumptions of the variance analysis.

\section{Results and Discussion}

\subsection{Crop Injury}

There was minimal visible injury at 1 and 4 WAE and no effect on seed moisture content (maturity) of white bean for the herbicide treatments evaluated (data not shown).

\subsection{Weed Control}

Dominant weeds in this study as determined by quantification and qualification of non-treated control plots included A. retroflexus (AMARE), A. artemisiifolia (AMBEL), C. album (CHEAL), S. viridis (SETVI), and S. arvensis (SINAR).

Pendimethalin provided $91 \%-93 \%, 13 \%-21 \%, 56 \%$ $92 \%, 96 \%-98 \%$, and $16 \%-24 \%$ control of AMARE, AMBEL, CHEAL, SETVI, and SINAR, respectively (Tables 1, 2).

Trifluralin provided $93 \%-99 \%, 34 \%-43 \%, 65 \%$ -
$99 \%, 95 \%-100 \%$, and $20 \%-47 \%$ control of AMARE, AMBEL, CHEAL, SETVI, and SINAR, respectively (Tables 1, 2). Trifluralin applied at 600 or $1155 \mathrm{~g} \cdot \mathrm{ai} \cdot \mathrm{ha}^{-1}$ provided similar weed control except CHEAL control at $4 \mathrm{WAE}$ and SETVI control at $8 \mathrm{WAE}$ which were greater at the higher rate.

EPTC provided $66 \%-77 \%, 61 \%-76 \%, 64 \%-86 \%$, $94 \%-99 \%$, and $52 \%-74 \%$ control of AMARE, AMBEL, CHEAL, SETVI, and SINAR, respectively (Tables 1, 2). EPTC applied at 3400 or $4400 \mathrm{~g} \cdot$ ai $\cdot \mathrm{ha}^{-1}$ provided similar weed control except CHEAL control at 8 WAE in 2009 which was higher at $4400 \mathrm{~g} \cdot \mathrm{ai} \cdot \mathrm{ha}^{-1}$.

S-metolachlor provided $92 \%-98 \%, 27 \%-48 \%, 28 \%$ $76 \%, 96 \%-99 \%$, and $33 \%-59 \%$ control of AMARE, AMBEL, CHEAL, SETVI, and SINAR, respectively (Tables 1, 2). S-metolachlor applied at 1050 or 1600 $\mathrm{g} \cdot \mathrm{ai} \cdot \mathrm{ha}^{-1}$ provided similar weed control except SINAR control at $4 \mathrm{WAE}$ which was higher at $1600 \mathrm{~g} \cdot \mathrm{ai} \cdot \mathrm{ha}^{-1}$.

Dimethenamid-p provided $98 \%-99 \%, 50 \%-72 \%$, $33 \%-88 \%, 98 \%-99 \%$, and $56 \%-78 \%$ control of AMARE, AMBEL, CHEAL, SETVI, and SINAR, respectively (Tables 1, 2). There was no difference between dimethenamid-p rates for the control of the weeds evaluated.

Imazethapyr provided $98 \%-100 \%, 72 \%-84 \%, 97 \%$ $100 \%, 77 \%-88 \%$, and $98 \%-100 \%$ control of AMARE, AMBEL, CHEAL, SETVI, and SINAR, respectively (Tables 1,2 ). There was no difference between imazethapyr rates for the control of the weeds evaluated.

In other studies, there was no difference between s-metolachlor and trifluralin applied PPI for the control of AMARE and SETVI [16]. However, trifluralin applied PPI provided better control of CHEAL ( $83 \%$ vs. $71 \%)$ compared with s-metolachlor [16]. Wall [17] reported improved control of AMARE and CHEAL when imazethapyr was applied at $50 \mathrm{~g} \cdot \mathrm{ha}^{-1}$ in white bean. Blackshaw and Esau [18] also found similar results in pinto bean. However, Cantwell et al. [19] reported that imazethapyr at $50 \mathrm{~g} \cdot \mathrm{ha}^{-1}$ provided only $30 \%$ control of CHEAL in soybean.

\subsection{Weed Density}

There was no significant difference in SINAR density with herbicides evaluated (Table 3). Pendimethalin caused as much as $91 \%, 21 \%, 81 \%$, and $91 \%$ reduction in density of AMARE, AMBEL, CHEAL, and SETVI (Table 3).

Trifluralin caused as much as $95 \%, 41 \%, 97 \%$, and 97\% reduction in density of AMARE, AMBEL, CHEAL, and SETVI, respectively (Table 3). Trifluralin applied at 600 or $1155 \mathrm{~g} \cdot \mathrm{ai} \cdot \mathrm{ha}^{-1}$ caused a similar decrease in density except for AMBEL and CHEAL density which were lower at the higher rate. 
Table 1. Visual estimates of percent weed control 4 WAE with various PPI herbicides in white bean at Exeter, ON from 2009 to 2011. Means followed by the same letter within a column are not significantly different according to Fisher's Protected LSD at $\mathbf{P}<0.05$.

\begin{tabular}{|c|c|c|c|c|c|c|c|c|c|c|c|}
\hline \multirow{3}{*}{$\begin{array}{c}\text { Treatment } \\
\text { Weedy check }\end{array}$} & \multirow{3}{*}{$\begin{array}{c}\begin{array}{c}\text { Rate } \\
\mathrm{g} \cdot \mathrm{ai} \cdot \mathrm{ha}^{-1}\end{array} \\
\end{array}$} & \multicolumn{10}{|c|}{ Weed control } \\
\hline & & \multicolumn{2}{|c|}{ AMARE } & \multicolumn{2}{|c|}{ AMBEL } & \multicolumn{2}{|c|}{ CHEAL } & \multicolumn{2}{|c|}{ SETVI } & \multicolumn{2}{|c|}{ SINAR } \\
\hline & & 0 & $\mathrm{f}$ & 0 & $\mathrm{f}$ & 0 & e & 0 & $\mathrm{e}$ & 0 & $\mathrm{~g}$ \\
\hline Weed-free check & & 100 & a & 100 & $\mathrm{a}$ & 100 & a & 100 & $\mathrm{a}$ & 100 & $\mathrm{a}$ \\
\hline Pendimethalin & 1080 & 91.0 & $\mathrm{~cd}$ & 13.3 & $\mathrm{f}$ & 89.3 & $\mathrm{~b}$ & 97.8 & $\mathrm{bc}$ & 15.8 & $\mathrm{f}$ \\
\hline Trifluralin & 600 & 97.6 & $a b c$ & 34.2 & $\mathrm{e}$ & 91.1 & $\mathrm{~b}$ & 96.8 & $\mathrm{c}$ & 44.2 & $\mathrm{e}$ \\
\hline Trifluralin & 1155 & 98.5 & $a b c$ & 38.8 & $\mathrm{e}$ & 99.0 & $\mathrm{a}$ & 98.7 & $a b c$ & 47.1 & de \\
\hline EPTC & 3400 & 72.8 & $\mathrm{e}$ & 71.2 & $\mathrm{bc}$ & 84.9 & $\mathrm{bc}$ & 99.0 & $a b c$ & 68.3 & $\mathrm{bc}$ \\
\hline EPTC & 4400 & 77.1 & de & 75.8 & $\mathrm{bc}$ & 85.1 & $\mathrm{bc}$ & 99.8 & $\mathrm{ab}$ & 70.8 & $\mathrm{bc}$ \\
\hline S-metolachlor & 1050 & 93.0 & bcd & 43.3 & $\mathrm{e}$ & 66.5 & $\mathrm{~d}$ & 99.0 & $a b c$ & 40.0 & $\mathrm{e}$ \\
\hline S-metolachlor & 1600 & 97.0 & $a b c$ & 47.9 & de & 75.9 & $\mathrm{~cd}$ & 99.6 & $a b$ & 58.8 & $\mathrm{~cd}$ \\
\hline Dimethenamid-p & 544 & 97.6 & $a b c$ & 60.0 & $\mathrm{~cd}$ & 84.0 & $\mathrm{bc}$ & 98.8 & $a b c$ & 65.8 & bc \\
\hline Dimethenamid-p & 693 & 97.6 & $a b c$ & 71.7 & $\mathrm{bc}$ & 88.3 & $\mathrm{bc}$ & 99.1 & $a b c$ & 78.3 & $\mathrm{~b}$ \\
\hline Imazethapyr & 45 & 98.7 & $a b c$ & 83.3 & $\mathrm{~b}$ & 99.1 & $\mathrm{a}$ & 81.3 & d & 98.5 & $\mathrm{a}$ \\
\hline Imazethapyr & 75 & 99.9 & $a b$ & 83.9 & $a b$ & 99.8 & $\mathrm{a}$ & 87.5 & d & 99.8 & $\mathrm{a}$ \\
\hline
\end{tabular}

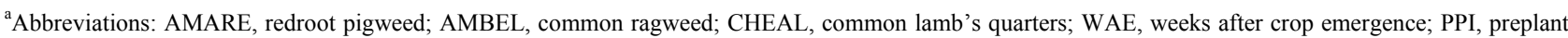
incorporated; SETVI, green foxtail; SINAR, wild mustard.

Table 2. Visual estimates of percent weed control 8 WAE with various PPI herbicides in white bean at Exeter, ON from 2009 to 2011. Means followed by the same letter within a column are not significantly different according to Fisher's Protected LSD at $\mathbf{P}<0.05^{\mathrm{a}}$.

\begin{tabular}{|c|c|c|c|c|c|c|c|c|c|c|c|c|c|}
\hline \multirow{5}{*}{$\begin{array}{c}\text { Treatment } \\
\text { Weedy check }\end{array}$} & \multirow{4}{*}{$\begin{array}{c}\text { Rate } \\
\mathrm{g} \cdot \mathrm{ai}^{\circ} \cdot \mathrm{ha}^{-1}\end{array}$} & \multicolumn{12}{|c|}{ Weed control } \\
\hline & & \multirow{2}{*}{\multicolumn{2}{|c|}{ AMARE }} & \multirow{2}{*}{\multicolumn{2}{|c|}{ AMBEL }} & \multicolumn{4}{|c|}{ CHEAL } & \multirow{2}{*}{\multicolumn{2}{|c|}{ SETVI }} & \multirow{2}{*}{\multicolumn{2}{|c|}{ SINAR }} \\
\hline & & & & & & \multicolumn{2}{|c|}{2009} & \multicolumn{2}{|c|}{ 2010-2011 } & & & & \\
\hline & & \multicolumn{12}{|c|}{$\%$} \\
\hline & & 0 & $\mathrm{e}$ & 0 & $\mathrm{~h}$ & 0 & $\mathrm{e}$ & 0 & $\mathrm{~d}$ & 0 & $\mathrm{e}$ & 0 & $\mathrm{f}$ \\
\hline Weed-free check & & 100 & $\mathrm{a}$ & 100 & $\mathrm{a}$ & 100 & $\mathrm{a}$ & 100 & $\mathrm{a}$ & 100 & $\mathrm{a}$ & 100 & $\mathrm{a}$ \\
\hline Pendimethalin $\mathrm{H} 20$ & 1080 & 92.8 & $\mathrm{bc}$ & 20.8 & gh & 56.3 & $\mathrm{c}$ & 91.5 & $\mathrm{a}$ & 96.2 & $\mathrm{c}$ & 24.2 & ef \\
\hline Trifluralin & 600 & 97.2 & $a b c$ & 34.2 & efg & 65.0 & $\mathrm{bc}$ & 87.5 & $a b$ & 94.5 & $\mathrm{c}$ & 19.6 & ef \\
\hline Trifluralin & 1155 & 99.6 & $\mathrm{ab}$ & 43.3 & def & 81.3 & $\mathrm{ab}$ & 94.6 & $\mathrm{a}$ & 99.6 & $a b$ & 30.0 & de \\
\hline EPTC & 3400 & 65.9 & $\mathrm{~d}$ & 60.8 & bcd & 65.0 & $\mathrm{bc}$ & 64.4 & $\mathrm{c}$ & 94.2 & $\mathrm{c}$ & 53.8 & $\mathrm{bc}$ \\
\hline ЕРTC & 4400 & 75.6 & $\mathrm{~d}$ & 65.0 & $\mathrm{bc}$ & 86.3 & $\mathrm{a}$ & 72.9 & $\mathrm{bc}$ & 96.5 & $\mathrm{c}$ & 52.1 & bcd \\
\hline S-metolachlor & 1050 & 91.9 & $\mathrm{c}$ & 26.7 & fgh & 27.5 & $\mathrm{~d}$ & 67.5 & $\mathrm{c}$ & 96.4 & $\mathrm{c}$ & 32.5 & cde \\
\hline S-metolachlor & 1600 & 97.6 & $a b c$ & 41.2 & ef & 44.6 & $\mathrm{~cd}$ & 68.8 & $\mathrm{c}$ & 96.2 & $\mathrm{c}$ & 51.7 & bcd \\
\hline Dimethenamid-p & 544 & 98.0 & $a b c$ & 49.6 & cde & 32.5 & $\mathrm{~d}$ & 72.5 & $\mathrm{bc}$ & 97.7 & $\mathrm{bc}$ & 56.2 & $\mathrm{~b}$ \\
\hline Dimethenamid-p & 693 & 98.6 & $a b c$ & 60.8 & bcd & 45.0 & $\mathrm{~cd}$ & 72.5 & $\mathrm{bc}$ & 96.8 & $\mathrm{bc}$ & 63.3 & $\mathrm{~b}$ \\
\hline Imazethapyr & 45 & 97.1 & $a b c$ & 72.1 & $\mathrm{~b}$ & 97.5 & $\mathrm{a}$ & 96.9 & $\mathrm{a}$ & 76.7 & $\mathrm{~d}$ & 97.9 & $\mathrm{a}$ \\
\hline Imazethapyr & 75 & 97.8 & $a b c$ & 78.3 & $\mathrm{~b}$ & 99.3 & $\mathrm{a}$ & 98.9 & $\mathrm{a}$ & 85.4 & $\mathrm{~d}$ & 100.0 & $\mathrm{a}$ \\
\hline
\end{tabular}

a Abbreviations: AMARE, redroot pigweed; AMBEL, common ragweed; CHEAL, common lamb's quarters; DAE, days after crop emergence; PPI, preplant incorporated; SETVI, green foxtail; SINAR, wild mustard. 
Table 3. Weed density 8 WAE with various PPI herbicides in white bean at Exeter, ON from 2009 to 2011. Means followed by the same letter within a column are not significantly different according to Fisher's Protected LSD at $\mathbf{P}<0.05^{\mathrm{a}}$.

\begin{tabular}{|c|c|c|c|c|c|c|c|c|c|c|c|c|c|}
\hline \multirow{4}{*}{ Treatment } & \multirow{4}{*}{$\begin{array}{c}\text { Rate } \\
\mathrm{g} \cdot \mathrm{ai}^{-} \mathrm{ha}^{-1}\end{array}$} & \multicolumn{12}{|c|}{ Weed density } \\
\hline & & \multirow{2}{*}{\multicolumn{2}{|c|}{ AMARE }} & \multirow{2}{*}{\multicolumn{2}{|c|}{ AMBEL }} & \multicolumn{4}{|c|}{ CHEAL } & \multirow{2}{*}{\multicolumn{2}{|c|}{ SETVI }} & \multirow{2}{*}{\multicolumn{2}{|c|}{ SINAR }} \\
\hline & & & & & & \multicolumn{2}{|c|}{2009} & \multicolumn{2}{|c|}{ 2010-2011 } & & & & \\
\hline & & \multicolumn{12}{|c|}{$\# \mathrm{~m}^{-2}$} \\
\hline Weedy check & & 20.3 & $\mathrm{e}$ & 8.5 & fgh & 97.0 & $f$ & 15.0 & d & 38.7 & $\mathrm{~d}$ & 13.9 & a \\
\hline Weed-free check & & 0 & a & 0 & a & 0 & $\mathrm{a}$ & 0 & $\mathrm{a}$ & 0 & $\mathrm{a}$ & 0 & a \\
\hline Pendimethalin $\mathrm{H} 20$ & 1080 & 1.8 & bcd & 6.7 & efgh & 20.8 & cde & 2.8 & $a b c$ & 3.6 & $\mathrm{bc}$ & 6.6 & a \\
\hline Trifluralin & 600 & 1.8 & bcd & 12.1 & $\mathrm{~h}$ & 27.2 & de & 1.8 & $a b c$ & 3.0 & $\mathrm{bc}$ & 11.0 & $\mathrm{a}$ \\
\hline Trifluralin & 1155 & 1.0 & abcd & 5.0 & defg & 9.7 & $\mathrm{c}$ & 0.4 & $\mathrm{a}$ & 1.3 & $a b$ & 7.2 & $\mathrm{a}$ \\
\hline EPTC & 3400 & 3.1 & $\mathrm{c}$ & 3.0 & bcd & 13.3 & $\mathrm{~cd}$ & 5.7 & $\mathrm{~cd}$ & 2.7 & $\mathrm{bc}$ & 3.5 & $\mathrm{a}$ \\
\hline EPTC & 4400 & 3.7 & $\mathrm{~d}$ & 3.6 & bcde & 14.1 & $\mathrm{~cd}$ & 4.9 & $\mathrm{~cd}$ & 2.6 & $\mathrm{bc}$ & 10.5 & $\mathrm{a}$ \\
\hline S-metolachlor & 1050 & 1.7 & bed & 9.3 & gh & 46.4 & ef & 4.2 & bcd & 2.4 & $\mathrm{bc}$ & 8.4 & a \\
\hline S-metolachlor & 1600 & 0.7 & $a b c$ & 4.6 & cdef & 27.7 & de & 4.2 & bcd & 2.1 & $\mathrm{bc}$ & 4.1 & a \\
\hline Dimethenamid-p & 544 & 0.8 & $a b c$ & 7.3 & efgh & 17.1 & $\mathrm{~cd}$ & 2.3 & $a b c$ & 1.1 & $a b$ & 3.0 & $\mathrm{a}$ \\
\hline Dimethenamid-p & 693 & 0.2 & $a b$ & 3.7 & cde & 28.1 & de & 1.7 & $a b c$ & 1.2 & $a b$ & 7.2 & a \\
\hline Imazethapyr & 45 & 0.2 & $a b$ & 2.2 & $a b c$ & 2.6 & $\mathrm{~b}$ & 0.5 & $a b$ & 6.9 & $\mathrm{c}$ & 0 & a \\
\hline Imazethapyr & 75 & 0.2 & $a b$ & 1.6 & $a b$ & 2.7 & $\mathrm{~b}$ & 0.2 & $\mathrm{a}$ & 6.6 & $\mathrm{c}$ & 0 & $\mathrm{a}$ \\
\hline
\end{tabular}

aAbbreviations: AMARE, redroot pigweed; AMBEL, common ragweed; CHEAL, common lamb's quarters; WAE, weeks after crop emergence; PPI, preplant incorporated; SETVI, green foxtail; SINAR, wild mustard.

EPTC provided $85 \%, 63 \%, 86 \%$, and $93 \%$ reduction in AMARE, AMBEL, CHEAL, and SETVI, respectively (Table 3). EPTC applied at 3400 or $4400 \mathrm{~g} \cdot \mathrm{ai} \cdot \mathrm{ha}^{-1}$ generally caused similar a similar decrease in density of the weeds evaluated.

S-metolachlor caused $97 \%, 46 \%, 72 \%$, and $95 \%$ reduction in density of AMARE, AMBEL, CHEAL, and SETVI, respectively (Table 3). S-metolachlor applied at 1050 or $1600 \mathrm{~g} \cdot \mathrm{ai} \cdot \mathrm{ha}^{-1}$ generally caused similar a similar decrease in density of the weeds evaluated.

Dimethenamid-p caused $99 \%, 56 \%, 89 \%$, and $97 \%$ reduction in density of AMARE, AMBEL, CHEAL, and SETVI, respectively (Table 3 ). There was no difference between dimethenamid-p rates in respect to weed density.

Imazethapyr caused $99 \%, 81 \%, 99 \%$, and $83 \%$ reduction in density of AMARE, AMBEL, CHEAL, and SETVI, respectively (Table 3 ). There was no difference between imazethapyr rates in respect to weed density.

\subsection{Weed Dry Weight}

Pendimethalin caused $95 \%, 18 \%, 81 \%, 87 \%, 93 \%$, and $51 \%$ reduction in dry weight of AMARE, AMBEL, CHEAL, SETVI, and SINAR, respectively (Table 4).

Trifluralin caused $98 \%, 61 \%, 99 \%, 98 \%$, and $77 \%$ reduction in dry weight of AMARE, AMBEL, CHEAL, SETVI, and SINAR, respectively (Table 4). Trifluralin

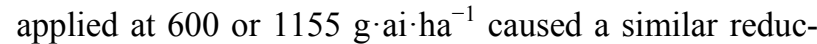
tion in dry weight of weeds evaluated.

EPTC caused $89 \%, 90 \%, 87 \%, 96 \%$, and $96 \%$ reduction in dry weight of AMARE, AMBEL, CHEAL, SETVI, and SINAR, respectively (Table 4). EPTC applied at 3400 or $4400 \mathrm{~g} \cdot \mathrm{ai} \cdot \mathrm{ha}^{-1}$ caused a similar reduction of weeds evaluated.

S-metolachlor caused $99 \%, 56 \%, 81 \%, 97 \%$, and $95 \%$ reduction in dry weight of AMARE, AMBEL, CHEAL, SETVI, and SINAR, respectively (Table 4). S-metolachlor applied at 1050 or $1600 \mathrm{~g} \cdot \mathrm{ai} \cdot \mathrm{ha}^{-1}$ caused a similar reduction in dry weight of weeds evaluated.

Dimethenamid-p caused $100 \%, 57 \%, 93 \%, 98 \%$, and $96 \%$ reduction in dry weight of AMARE, AMBEL, CHEAL, SETVI, and SINAR, respectively (Table 4). There was no difference between dimethenamid-p rates in respect to weed dry weight reduction.

Imazethapyr caused 100\%, 95\%, 100\%, 99\%, 92\%, and $100 \%$ reduction in dry weight of AMARE, AMBEL, CHEAL, SETVI, and SINAR, respectively (Table 4). There was no difference between the imazethapyr rates evaluated in respect to weed dry weight reduction.

\subsection{Yield}

Pendimethalin, trifluralin, EPTC, s-metolachlor, dimethenamid-p, and imazethapyr applied preplant incorporated increased yield of white bean as much as $35 \%$ - 
Table 4. Weed dry weight 8 WAE with various PPI herbicides in white bean at Exeter, ON from 2009 to 2011 . Means followed by the same letter within a column are not significantly different according to Fisher's Protected LSD at $\mathbf{P}<0.05^{\mathrm{a}}$.

\begin{tabular}{|c|c|c|c|c|c|c|c|c|c|c|c|c|c|}
\hline \multirow{4}{*}{ Treatment } & \multirow{4}{*}{$\begin{array}{c}\text { Rate } \\
\mathrm{g} \cdot \mathrm{ai} \cdot \mathrm{ha}^{-1}\end{array}$} & \multicolumn{12}{|c|}{ Weed dry weight } \\
\hline & & \multirow{2}{*}{\multicolumn{2}{|c|}{ AMARE }} & \multirow{2}{*}{\multicolumn{2}{|c|}{ AMBEL }} & \multicolumn{4}{|c|}{ CHEAL } & \multirow{2}{*}{\multicolumn{2}{|c|}{ SETVI }} & \multirow{2}{*}{\multicolumn{2}{|c|}{ SINAR }} \\
\hline & & & & & & \multicolumn{2}{|c|}{2009} & \multicolumn{2}{|c|}{ 2010-2011 } & & & & \\
\hline & & \multicolumn{12}{|c|}{$\mathrm{g} \cdot \mathrm{m}^{-2}$} \\
\hline Weedy check & & 44.6 & g & 36.9 & $\mathrm{~d}$ & 169.3 & $\mathrm{f}$ & 19.6 & $\mathrm{~d}$ & 31.5 & $\mathrm{c}$ & 235.4 & $\mathrm{c}$ \\
\hline Weed-free check & & 0 & $\mathrm{a}$ & 0 & a & 0 & a & 0 & a & 0 & $\mathrm{a}$ & 0 & $\mathrm{a}$ \\
\hline Pendimethalin H20 & 1080 & 2.4 & def & 30.1 & $\mathrm{~d}$ & 31.9 & cde & 2.5 & $a b c$ & 2.1 & $\mathrm{~b}$ & 115.1 & $\mathrm{bc}$ \\
\hline Trifluralin & 600 & 1.3 & bcde & 20.6 & d & 25.1 & bcd & 2.0 & $a b c$ & 1.7 & $\mathrm{ab}$ & 147.1 & $\mathrm{bc}$ \\
\hline Trifluralin & 1155 & 0.7 & abcd & 14.4 & bcd & 5.4 & $\mathrm{~b}$ & 0.2 & $\mathrm{ab}$ & 0.5 & $a b$ & 53.1 & $\mathrm{bc}$ \\
\hline EPTC & 3400 & 6.2 & $\mathrm{f}$ & 3.6 & $a b$ & 9.9 & $\mathrm{bc}$ & 4.9 & $\mathrm{~cd}$ & 1.6 & $\mathrm{ab}$ & 8.9 & $a b c$ \\
\hline EPTC & 4400 & 4.8 & ef & 6.0 & $a b c$ & 11.0 & $\mathrm{bc}$ & 2.6 & $a b c$ & 1.4 & $\mathrm{ab}$ & 25.2 & $\mathrm{bc}$ \\
\hline S-metolachlor & 1050 & 1.5 & bcde & 35.6 & d & 127.7 & ef & 6.0 & $\mathrm{~cd}$ & 0.8 & $a b$ & 48.4 & $\mathrm{bc}$ \\
\hline S-metolachlor & 1600 & 0.6 & abcd & 16.1 & $\mathrm{~cd}$ & 93.3 & def & 3.7 & $\mathrm{bc}$ & 1.8 & $\mathrm{ab}$ & 11.6 & $a b c$ \\
\hline Dimethenamid-p & 544 & 2.0 & cdef & 22.1 & d & 71.7 & def & 3.2 & $a b c$ & 0.5 & $a b$ & 8.4 & $a b$ \\
\hline Dimethenamid-p & 693 & 0.2 & $a b c$ & 15.8 & $\mathrm{~cd}$ & 111.8 & def & 1.3 & $a b c$ & 1.0 & $\mathrm{ab}$ & 21.2 & bc \\
\hline Imazethapyr & 45 & 0.1 & $a b$ & 2.0 & $a b$ & 0.3 & a & 0.2 & $\mathrm{ab}$ & 3.1 & $\mathrm{~b}$ & 0 & $\mathrm{a}$ \\
\hline Imazethapyr & 75 & 0.3 & abcd & 2.1 & $a b$ & 0.2 & $\mathrm{a}$ & 0.1 & $a b$ & 2.4 & $\mathrm{~b}$ & 0 & $\mathrm{a}$ \\
\hline
\end{tabular}

aAbbreviations: AMARE, redroot pigweed; AMBEL, common ragweed; CHEAL, common lamb's quarters; WAE, weeks after crop emergence; PPI, preplant incorporated; SETVI, green foxtail; SINAR, wild mustard.

$46 \%, 49 \%$ - 72\%, 37\% - 248\%, 44\% - 102\%, 42\% $217 \%$, and $37 \%-426 \%$ compared to the weedy check, respectively (Table 5). However, only imazethapyr applied PPI at the $75 \mathrm{~g} \cdot \mathrm{ai} \cdot \mathrm{ha}^{-1}$ provided yield equivalent to the weed-free check in 2009 and 2011 (Table 5). All other herbicide treatments had significantly lower yield than the weed-free check. All herbicide treatments except pendimethalin at $1080 \mathrm{~g} \cdot \mathrm{ai} \cdot \mathrm{ha}^{-1}, \mathrm{EPTC}$ at $4400 \mathrm{~g} \cdot \mathrm{ai}^{-\mathrm{ha}^{-1}}{ }^{-1}$ and imazethapyr at $75 \mathrm{~g} \cdot \mathrm{ai} \cdot \mathrm{ha}^{-1}$ had yield equivalent to the weed-free check in 2010 (Table 5). Other studies have shown yield losses of $40 \%-71 \%$ in white bean when broadleaf weeds such as AMARE and CHEAL were not adequately controlled [17]. Blackshaw and Esau [18] also reported $71 \%-85 \%$ yield losses in pinto bean when AMARE and CHEAL were left uncontrolled.

\subsection{Environmental Impact}

The EI calculated based on Kovach [11] are listed in Table 5. The EI values ranged from 0.8 to 36.9 depending on the herbicide and rate evaluated. Based on the EI analysis, the herbicide program which resulted in the lowest environmental risk was imazethapyr (0.8 and 1.3) followed by dimethenamid-p (5.8 and 7.4), trifluralin (10.1 and 19.4), s-metolachlor (20.6 and 31.4), EPTC (28.5 and 36.9), and then pendimethalin (29.1). Lower rates of each herbicide resulted in a lower EI as expected
(Table 5).

\subsection{Profitability Analysis}

In 2009 and 2011, imazethapyr applied PPI at $45 \mathrm{~g} \cdot$ ai $\cdot \mathrm{ha}^{-1}$ had a profit margin of $\$ 1825.41$ and when applied at 75 $\mathrm{g} \cdot \mathrm{ai} \cdot \mathrm{ha}^{-1}$ had a profit margins $\$ 1912.97$ which was significantly greater than dimethenamid-p and EPTC which at best had a profit margin of $\$ 1166.26$. Profit margin with other herbicide treatments such as s-metolachlor, trifluralin, and pendimethalin were significantly lower and ranged from $\$ 503.51$ to $\$ 660.41$ (Table 5). In 2010, there was no difference in profit margin among the herbicides evaluated (Table 5).

\section{Conclusion}

Based on this study, there was minimal visible injury with pendimethalin, trifluralin, EPTC, s-metolachlor, dimethenamid-p, and imazethapyr applied PPI in white bean. The control of AMARE, AMBEL, CHEAL, SETVI, and SINAR were as much as 93\%, 21\%, 92\%, 98\%, and $24 \%$ with pendimethalin, 99\%, 43\%, 99\%, 100\%, and $47 \%$ with trifluralin, $77 \%, 76 \%, 86 \%, 99 \%$, and $74 \%$ with EPTC, $98 \%, 48 \%, 76 \%, 99 \%$, and $59 \%$ with s-metolachlor, 99\%, 72\%, 88\%, 99\%, and 78\% with dimethenamid, and $100 \%, 84 \%, 100 \%, 88 \%$, and $100 \%$ with imazethapyr, respectively. Weed density and dry 
Table 5. White bean yield and profit margin for various PPI herbicide treatments at Exeter, ON from 2009 to 2011 . Means followed by the same letter within a column are not significantly different according to Fisher's Protected LSD at $\mathbf{P}<0.05$.

\begin{tabular}{|c|c|c|c|c|c|c|c|c|c|c|}
\hline \multirow{4}{*}{$\begin{array}{c}\text { Treatment } \\
\text { Weedy check }\end{array}$} & \multirow{4}{*}{$\begin{array}{c}\text { Rate } \\
\mathrm{g} \cdot \text { ai }^{-h^{-1}}\end{array}$} & \multicolumn{4}{|c|}{ Yield } & \multirow{4}{*}{$\mathbf{E I}^{\mathrm{a}}$} & \multicolumn{4}{|c|}{ Profit Margin } \\
\hline & & \multicolumn{2}{|c|}{ 2009, 2011} & \multicolumn{2}{|c|}{2010} & & \multicolumn{2}{|c|}{ 2009, 2011} & \multicolumn{2}{|c|}{2010} \\
\hline & & \multicolumn{4}{|c|}{ MT ha $^{-1}$} & & \multicolumn{4}{|c|}{$\$ \mathrm{ha}^{-1}$} \\
\hline & & 0.46 & $\mathrm{f}$ & 2.18 & $\mathrm{~d}$ & & & & & \\
\hline Weed-free check & & 2.82 & a & 3.38 & $\mathrm{a}$ & & & & & \\
\hline Trifluralin & 600 & 0.79 & ef & 3.24 & $\mathrm{abc}$ & 10.1 & 573.05 & $\mathrm{~cd}$ & 2155.73 & $\mathrm{a}$ \\
\hline Trifluralin & 1155 & 0.69 & ef & 3.25 & $\mathrm{abc}$ & 19.4 & 518.77 & $\mathrm{~d}$ & 2148.37 & $\mathrm{a}$ \\
\hline EPTC & 3400 & 1.32 & $\mathrm{~cd}$ & 3.20 & $a b c$ & 28.5 & 980.42 & $\mathrm{~b}$ & 2072.55 & $\mathrm{a}$ \\
\hline ЕРТC & 4400 & 1.60 & c & 2.98 & $\mathrm{bc}$ & 36.9 & 1166.26 & $\mathrm{~b}$ & 1904.38 & $\mathrm{a}$ \\
\hline S-metolachlor & 1600 & 0.93 & def & 3.25 & $a b c$ & 31.4 & 660.41 & $\mathrm{~cd}$ & 2117.45 & $\mathrm{a}$ \\
\hline Dimethenamid-p & 544 & 1.13 & cde & 3.36 & $\mathrm{ab}$ & 5.8 & 833.86 & $\mathrm{bc}$ & 2205.74 & $\mathrm{a}$ \\
\hline Dimethenamid-p & 693 & 1.46 & $\mathrm{c}$ & 3.10 & $a b c$ & 7.4 & 1096.88 & $\mathrm{~b}$ & 2022.82 & $\mathrm{a}$ \\
\hline Imazethapyr & 45 & 2.27 & $\mathrm{~b}$ & 3.13 & $\mathrm{abc}$ & 0.8 & 1825.41 & $\mathrm{a}$ & 2058.18 & $\mathrm{a}$ \\
\hline Imazethapyr & 75 & 2.42 & $a b$ & 2.98 & $\mathrm{bc}$ & 1.3 & 1912.97 & $\mathrm{a}$ & 1928.23 & $\mathrm{a}$ \\
\hline
\end{tabular}

${ }^{\mathrm{a}}$ The EI calculated based on Kovach's environmental impact quotient (EIQ) [11].

weight reductions were similar to visible weed control. Imazethapyr applied PPI at the $75 \mathrm{~g} \cdot \mathrm{ai}^{\mathrm{a}} \cdot \mathrm{ha}^{-1}$ provided yield equivalent to weed-free check in 2009 and 2011. All other herbicide treatments had significantly lower yield than the weed-free check. All herbicide treatments except pendimethalin at $1080 \mathrm{~g} \cdot \mathrm{ai} \cdot \mathrm{ha}^{-1}, \mathrm{EPTC}$ at $4400 \mathrm{~g} \cdot \mathrm{ai} \cdot \mathrm{ha}^{-1}$, and imazethapyr at $75 \mathrm{~g} \cdot \mathrm{ai} \cdot \mathrm{ha}^{-1}$ had yield equivalent to the weed-free check in 2010. Based on EI analysis, the herbicide program which resulted in the lowest environmental risk was imazethapyr followed by dimethenamid-p, trifluralin, s-metolachlor, EPTC, and then pendimethalin. Lower rates of each herbicide provided lower risks to the environment as expected. Economic analysis of herbicide treatments evaluated indicates that imazethapyr had the greatest positive impact on profit margins, followed by dimethenamid-p and EPTC, followed by s-metolachlor and trifluralin and then pendimethalin.

\section{Acknowledgements}

The authors would like to acknowledge Todd Cowan for his expertise and technical assistance in these studies. Funding for this project was provided by the Ontario White Bean Producers and the Agricultural Adaptation Council.

\section{REFERENCES}

[1] T. Breuer, "The Emerging Bean, Harvest 2002 Edition," Ontario White Bean Producers, London, 2002, 16 p.
[2] B. McGee, "Estimated Area, Yield, Production and Farm Value of Specified Field Crops, Ontario, 2001-2011." http://www.omafra.gov.on.ca/english/stats/crops/estimate _metric.htm

[3] D. Chikoye, S. F. Weise and C. J. Swanton, "Influence of Common Ragweed (Ambrosia artemisiifolia) Time of Emergence and Density on White Bean (Phaseolus vulgaris)," Weed Science, Vol. 43, No. 3, 1995, pp. 375-380.

[4] V. S. Malik, C. J. Swanton and T. E. Michaels, "Interaction of White Bean (Phaseolus vulgaris) Cultivars, Row Spacing, and Seeding Density with Annual Weeds," Weed Science, Vol. 41, No. 1, 1993, pp. 62-68.

[5] C. P. Urwin, R. G. Wilson and D. A. Mortensen, "Responses of Dry Edible Bean (Phaseolus vulgaris) Cultivars to Four Herbicides," Weed Technology, Vol. 10, No. 3, 1996, pp. 512-518.

[6] Ontario Ministry of Agriculture, Food and Rural Affairs (OMAFRA), "Guide to Weed Control," Publication 75, Toronto, 2011, $348 \mathrm{p}$.

[7] S. A. Senseman, "Herbicide Handbook," Ninth Edition. Champaign, Weed Science Society of America, 2007, 458 p.

[8] N. R. Arnold, W. M. Murray, J. E. Gregory and D. Smeal, "Weed Control in Pinto Beans (Phaseolus vulgaris) with Imazethapyr Combinations," Weed Technology, Vol. 7, No. 2, 1993, pp. 361-364.

[9] T. A. Bauer, K. A. Renner, D. Penner and J. D. Kelly, "Pinto Bean (Phaseolus vulgaris) Varietal Tolerance to Imazethapyr," Weed Science, Vol. 43, No. 3, 1995, pp. 417-424.

[10] R. G. Wilson and S. D. Miller, "Dry Edible Bean (Phase- 
olus vulgaris) Responses to Imazethapyr," Weed Technology, Vol. 5, No. 1, 1991, pp. 22-26.

[11] J. Kovach, C. Petzoldt, J. Degni and J. Tette, "A Method to Measure the Environmental Impact of Pesticides," New York's Food Life Sciences Bulletin, Vol. 139, 1992, pp. 139-146 (Updated 2004).

[12] T. A. Brimner, G. J. Gallivan and G. R. Stephenson, "Influence of Herbicide-Resistant Canola on the Environmental Impact of Weed Management," Pest Management Science, Vol. 61, No. 1, 2005, pp. 47-52. doi:10.1002/ps.967

[13] N. Soltani, L. L. Van Eerd, R. J. Vyn, C. Shropshire and P. H. Sikkema, "Weed Management in Dry Bean (Phaseolus vulgaris) with Dimethenamid plus Reduced Doses of Imazethapyr Applied Preplant Incorporated," Crop Protection, Vol. 26, No. 5, 2007, pp. 739-745. doi:10.1016/j.cropro.2006.06.013

[14] P. H. Sikkema, L. L. Van Eerd, R. Vyn and S. E. Weaver, "A Comparison of Reduced Rate and Economic Thresh- old Approaches to Weed Management in a Corn-Soybean Rotation," Weed Technology, Vol. 21, No. 3, 2007, pp. 647-655. doi:10.1614/WT-06-135.1

[15] AGRIS Co-Operative Ltd., "Chemical Prices 2009-2011," Chatham, 2009-2011.

[16] P. H. Sikkema, R. J. Vyn, C. Shropshire and N. Soltani, "Integrated Weed Management in White Bean Production," Canadian Journal of Plant Science, Vol. 88, No. 3, 2008, pp. 555-561. doi:10.4141/CJPS07180

[17] D. A. Wall, "Bentazon Tank Mixtures for Control of Redroot Pigweed and Common Lambsquarters in Navy Bean," Weed Technology, Vol. 9, 1995, pp. 610-616.

[18] R. E. Blackshaw and R. Esau, "Control of Annual Broadleaf Weeds in Pinto Beans (Phaseolus vulgaris)," Weed Technology, Vol. 5, 1991, pp. 532-538.

[19] J. R. Cantwell, R. A. Liebl and F. W. Slife, "Imazethapyr for Weed Control in Soybean (Glycine max)," Weed Technology, Vol. 3, 1991, pp. 596-601. 